\title{
Medicinal uses, phytochemistry and pharmacology of Ammodaucus leucotrichus
}

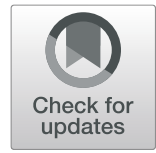

Elhassan Idm'hand ${ }^{*}$ (D), Fouad Msanda and Khalil Cherifi

\begin{abstract}
This review summarizes selected scientific evidence on phytochemistry and pharmacological properties of Ammodaucus leucotrichus. Information was gathered via the Internet (using Scopus, PubMed, Google Scholar, Elsevier, Springer, Science Direct, Researchgate and Web of Science) as well as from libraries and local books. Ammodaucus leucotrichus (A. leucotrichus), belongs to the family Apiaceae, has been reported to possess a wide range of traditional medicinal uses including in diarrhea, cough, pulmonary diseases, anorexia, allergy, tachycardia, helminthiasis, stomach pain, gastralgias, otitis, indigestion, cold, fever, anorexia and cardiac diseases. Phytochemical investigations revealed that this plant possesses many bioactive chemical constituents including monoterpenes and their derivatives, sesquiterpenes and their derivatives, tannins, anthracenes compounds, sterols, triterpenes, reducing compounds, alkaloids, phenol acids, saponins, flavonoids and coumarins. The most important pharmacological activities are antioxidant, antibacterial, antifungal, antidiabetic, anti-inflammatory, anticholinesterase and cytotoxicity activities. Ammodaucus leucotrichus has potential for the treatment of a wide range of diseases and has been well studied for its phytochemical properties. However, further scientific studies are needed to explore mechanisms of actions, adverse effects of the extracts, toxicity and the therapeutic effect of major secondary metabolites.
\end{abstract}

Keywords: Phytochemistry, Pharmacology, Medicinal, Biological activity, Ammodaucus leucotrichus

\section{Background}

The flora of Morocco with its 7000 species and subspecies belonging to several botanical families is not widely explored in the pharmacological aspects as well as in the phytochemical characteristics [1]. Therefore, the valorization of the medicinal plants of the Moroccan flora will be a great contribution to the pharmaceutical industry for selecting plants with potential pharmaceutical properties.

The Apiaceae (Umbelliferae), in the order Apiales, is one of the largest families with about 300 genera and more than 2500 species, distributed in a variety of habitats, principally in the temperate regions of the world [2, 3]. Ammodaucus leucotrichus Coss. \& Dur. (=Cuminum maroccanum P.H.Davis \& Hedge) is the only specie of the genus Ammodaucus in the Apiaceae family is referred to as 'Kamune es sufi or akâman' in most of the north African countries, while in Algeria

\footnotetext{
* Correspondence: idmhand-h@hotmail.com

Laboratoire de Biotechnologies et Valorisation des Ressources Naturelles Faculté des Sciences, B.P. 8106, Cité Dakhla, Agadir, Morocco
}

it is commonly known as "Moudrayga" and known as "Cumin chevelu" in French [4, 5]. It inhabits the maritime sands of the Saharan and sub-Saharan countries of North Africa, including Morocco, Algeria and Tunisia extending to Egypt and the tropical Africa. Its best implantation is in desert regions, often down a hill or a dune $[5,6]$. It is a small glabrous annual plant with finely striated stems, branched from the base (Fig. 1). The leaves are fleshy, finely divided forming flat narrow ridges with sheathing petioles. The white flowers, with five free petals, are grouped in composed umbels of two to four branches. The fruit is a di-achene with $8-10 \mathrm{~mm}$ long and is covered with very dense and fuzzy hairs $[2,5]$. This plant plays a major role in herbal medicine in North African countries.

Current paper aims to provide an overview on the ethnobotanical uses along with recent studies concerning the ethnopharmacology, phytochemistry and therapeutic activities of Ammodaucus leucotrichus. 


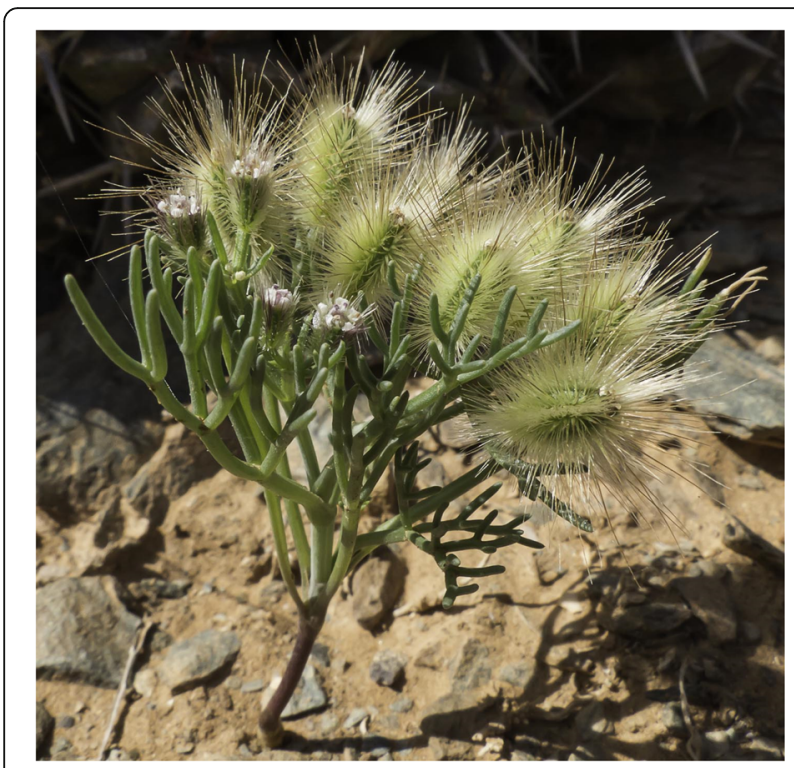

Fig. 1 Ammodaucus leucotrichus in natural habitat

\section{Traditional uses}

Ammodaucus leucotrichus is used in the North African countries as a condiment or spice and for varied medical applications [6].

In Morocco, different parts of this plant, especially fruits and seeds, are often used to treat gastricintestinal pains, gastralgias, otitis, hypotensive, indigestion, cold, fever, pulmonary diseases, labor pains, anorexia and cardiac diseases [3]. The fruits, in infusion, are used in various infantile diseases of the digestive system. They are also mixed with Thymus satureioides to treat stomach pains [7]. The decoction of flowers and seeds is used for abdominal pain for children and adults. The fruits in infusion are indicated to treat intestinal pain for infants [8]. Seeds prepared as soup or infusion, are used to soothe abdominal pain, nausea, vomiting in newborns. The infusion is recommended to help the elderly for sleeping [9]. A preparation based on the seeds of Ammodaucus leucotrichus and those of Petroselinum sativum, powdered and mixed with apple vinegar and pure honey, is used for cystitis and nephritic colics. The patient takes a teaspoon of the mix in a glass of hot water on an empty stomach. A preparation based on the seeds of Ammodaucus leucotrichus, Zea mays style, Opuntica ficus-indica flowers and Zizyphus lotus fruits, decocted in water, are used for pyelonephritis. The patient drinks two glasses everyday [10]. The seeds of Ammodaucus leucotrichus, powder of Myristica fragrans, Euphorbia falcata (1 spoon), Herniaria hirsuta (1 spoon), a lemon fruit and a cup of olive oil, decocted in water are used for the treatment of kidney stones [11]. The decoction of fruits and seeds is also used for diarrhea, emetic, cough, pulmonary diseases, anorexia, allergy, tachycardia, pains of tiredness, helminthiasis and gastroenteritis [12].

Ammodaucus leucotrichus, known in Algeria as "Moudrayga or El massoufa", is a medicinal plant sold to herbalists in local markets, particularly in the Southern Algerian Sahara. The nomads collect the seeds and leaves for their own use, usually in the spring, when the fruits are ripe [13]. In the southern Algerian Sahara, the leaves of this plant are used as a flavoring herbal in teas and fruits are often used as a spice during culinary preparation. The leaves and seeds are consumed in the form of decoction or infusion for several therapeutic cases, such as blood pressure, chest pain, liver and digestive system ailments, gastroenteritis, as also for diabetes [14]. In Tassili (Algeria), the fruits and the leaves are commonly consumed in infusion. The powder form is also an appreciated spice for foodstuff. The leaves consumed for their flavoring characteristics in tea, and the fruits for their bioactive capacity mainly in the treatment of heart palpitations [15]. The fruits of this plant are widely used as sugar regulator for diabetics and for stomach and colon diseases. The seeds are used to treat stomach diseases, wounds infections, cutaneous allergies, genital disorders, abdominal pains, for scorpion stings, snakebites, and liver diseases. The leaves are used infused or used as a powder to avoid indigestion, to recover the appetite and for chest complaints [3]. Ammodaucus leucotrichus is also frequently used in the treatment of allergy symptoms, diarrhea, indigestion, vomiting, spasms and colic, intestinal worms, fever, constipation, coughing and for anorexia [15]. Moreover, aphrodisiac, emmenagogue and abortive properties are attributed to this species [16].

\section{Phytochemistry}

Various bioactive compounds are isolated from aqueous, acetone, methanolic, or ethanolic extracts of the plant. They are grouped as monoterpenes and their derivatives, sesquiterpenes and their derivatives, tannins, anthracenes compounds, sterols, triterpenes, reducing compounds, alkaloids, phenol acids, saponins, flavonoids and coumarins [5]. In addition, reports on the isolation, and identification of individual chemical constituents have revealed the presence of perillaldehyde and limonene as major compounds. The new guainolide lactone, ammolactone and new monoterpenoid 3-hydroxyperiilaldehyde, methylperillate, borneolangelate and $\gamma$-decalactone were also found in this species $[2,17]$. The presence of such metabolites indicates the importance of plant extracts, for examples terpenes are considered as suitable penetration enhancers in pharmaceutical industries [18], alkaloids are considered to be the primary active ingredients in plant-derived medicines [19], flavonoids are considered important due to 
their antioxidant, anti-inflammatory, antimicrobial, anticancer, anti-HIV, anticoagulant, immunomodulatory, antitubercular and anti-allergic activities [20], Perillaldehyde, the major compound in Ammodaucus leucotrichus extracts, has the potential to conserve the quality and safety of foods and fresh produce. Additionally, perillaldehyde is widely used in flavour, cosmetics, perfumery, and pharmaceutical industry [3]. Table 1 shows the isolated phytochemicals from different parts of the plant in detail.

\section{Pharmacological activities}

Ammodaucus leucotrichus has been studied regarding their bioactive characteristics. Several extracts and isolated compounds have been evaluated for their biological activities, namely, antioxidant, antibacterial, antifungal, antidiabetic, anti-inflammatory, anticholinesterase and cytotoxicity activities.

\section{Antioxidant activities}

Louail et al. [25] assessed antioxidant activities of essential oil of seeds of Ammodaucus leucotrichus by measuring the inhibition of conjugated diene hydroperoxides arising from linoleic acid oxidation. It appeared that the essential oil obtained from the seeds of Ammodaucus leucotrichus demonstrated antioxidant activity, by inhibiting the $\beta$-carotene bleaching due to retarding/inhibiting of linoleic acid hyderoperoxide-derived, which attack the chromophore- $\beta$-carotene. The value of the essential oil of seeds showed a better antioxidant activity than that shown by Ascorbic acid. In another experiment, Dahmane et al. [17] assessed antioxidant activities of essential oils obtained from the fruits of Ammodaucus leucotrichus using 2, 2-diphenyl-1-picrylhydrazyl (DPPH) assay and the $\beta$-carotene bleaching assay. Essential oils of Ammodaucus leucotrichus exhibited weak antioxidant abilities to reduce DPPH radicals. When compared to BHT and ascorbic acid, oils have been found significantly less effective than these antioxidant agents $(p<$ 0.05). However, essential oils exhibited significantly lower inhibition against linoleic acid oxidation and weak antioxidant abilities for reduce DPPH radicals [17].

Sebaa et al. [5] evaluated antioxidant activities of oils essential, aqueous, and methanol extracts of fruits of Ammodaucus leucotrichus using the reducing power method and the DPPH method. The result of antioxidant activity by the ferric reducing was detected in all the extracts: weak in the aqueous extract, oils essential and strong in the extract methanolic of Ammodaucus leucotrichus fruits. Free radical scavenging activity of all the extracts and references antioxidants increased with the increase in concentration. Antioxidant activity dosing by using DPPH whitening assesses that methanol extract has the best performance. All extracts have a free radical reduction capacity. Required concentrations to neutralize and stabilize $50 \%$ of DPPH concentration goes from 2 to $4 \mu \mathrm{g} / \mathrm{ml}$ [5]. A potential antioxidant effect of the aqueous extract from the fruits of Ammodaucus leucotrichus has been shown, in vitro, by the method of trapping of free radical 2,2-diphenyl-1 picrylhydrazyl [26].

\section{Antimicrobial activities}

The essential oil of seeds of Ammodaucus leucotrichus was screened for its antimicrobial activity against different microorganisms, including Gram-positive bacteria Bacillus subtilis, Gram-negative bacteria Escherichia coli and two yeast species Candida albicans and Saccharomyces cerevisiae, in addition Aspergillus flavus and Penicillium expansum. Ammodaucus leucotrichus oil exhibited strong inhibitory action against most tested organisms with MIC values ranged from 0.37 to $0.92 \mathrm{mg} / \mathrm{ml}$. Furthermore, the essential oil showed significant antibacterial activity against Gram negative and Gram positive bacteria at doses of $1.29 \mathrm{mg} /$ disc. The antibacterial activity of essential oils also tested for yeast such as Candida albicans and Saccharomyces cerevisiae showed moderate to good activity. The antifungal results revealed good, clear zones of growth inhibition against Aspergillus flavus and Penicillium expansum [25].

Dahmane et al. [17] evaluated antibacterial activities of the essential oil acquired from the fruits of Ammodaucus leucotrichus using disc diffusion and agar dilution methods against nine bacterial strains, 5 Gram positive and 4 Gram negative. The essential oils exerted a broad antibacterial spectrum with diameter of inhibition zones ranging from 9.66 to $52.66 \mathrm{~mm}$, while it was $10.66-39.00 \mathrm{~mm}$ for the positive control.

Mbanga et al. Sebaa et al. [5] assessed antimicrobial activities of different fractions and extracts of Ammodaucus leucotrichus fruits against eight bacteria and three fungi strains: Escherichia coli, Pseudomonas aeruginosa, Klebsilla pneumonia, Salmonella typhimurium, Proteus vulgaris, Bacillus cereus, Staphylococcus aureus, Enterococcus faecalis, Candida albicans, Aspergillus niger and Trichophyton rubrum using the disk diffusion method. The inhibition zones of disc for strains were in the ranges $7.0-18.0 \mathrm{~mm}$. Generally, the extracts of the plant were moderately active against Gram positive and negative bacteria. All the extracts and fractions from Ammodaucus leucotrichus announced an antifungal activity and the greatest effect was obtained by butanic fraction on Trichophyton rubrum and methanolic extract on Candida albicans whose zones of inhibition were of 20 and $15 \mathrm{~mm}$ respectively [5].

The essential oil obtained from the seeds of Ammodaucus leucotrichus showed significant antibacterial activity against Micrococcus luteus while moderately active against Klebsiella pneumonia, Staphylococcus 
Table 1 Phytochemicals isolated from different parts of Ammodaucus leucotrichus

\begin{tabular}{|c|c|c|c|}
\hline No. & Chemical compound & Part of plant & References \\
\hline 1 & (+)-limonene & Seed & {$[21]$} \\
\hline 2 & (+)-perillaldehyde & Seed & {$[21]$} \\
\hline 3 & (+)-3-hydroxyperillaldehyd & Seed & {$[21]$} \\
\hline 4 & a-fenchene & Fruit & {$[22]$} \\
\hline 5 & Cis-Limonene oxide & Fruit & {$[3,17]$} \\
\hline 6 & 8-2-Carene & Seed & [16] \\
\hline 7 & $\beta$-Phellandrene & Seed, fruit & {$[3,6,16]$} \\
\hline 8 & cis-Ocimene & Seed & [16] \\
\hline 9 & Linalool & Seed, fruit & {$[6,16]$} \\
\hline 10 & $\gamma$-Terpineol & Seed & [16] \\
\hline 11 & Myrtenal & Seed, fruit & {$[6,16,17]$} \\
\hline 12 & cis-Carveol & Seed, fruit & {$[16,17]$} \\
\hline 13 & a-Terpinene-7-al & Seed & [16] \\
\hline 14 & Piperitenone & Seed & {$[16]$} \\
\hline 15 & $\beta$-Myrcene & Seed, fruit & {$[13,23]$} \\
\hline 16 & 3-Carene & Seed, fruit, aerial part & {$[3,4,13,15,23,24]$} \\
\hline 17 & Borneol & Seed, fruit & {$[6,13]$} \\
\hline 18 & a-Phellandrene & Fruit & {$[6,17]$} \\
\hline 19 & a-Terpinene & Fruit & {$[6]$} \\
\hline 20 & p-Cymene & Fruit & {$[6,17]$} \\
\hline 21 & cis-Sabinene hydrate & Fruit & {$[6]$} \\
\hline 22 & Camphor & Fruit & {$[3,6,17]$} \\
\hline 23 & p-Cymen-8-ol & Fruit & [6] \\
\hline 24 & Thymol methyl ether & Fruit & {$[6]$} \\
\hline 25 & a-Thujene & Seed, fruit & {$[17,25]$} \\
\hline 26 & a-Pinene & Seed, fruit, aerial part & {$[4,6,13,15-17,24,25]$} \\
\hline 27 & Camphene & Seed, fruit, aerial part & {$[6,13,15,17,24,25]$} \\
\hline 28 & Sabinene & Seed, fruit & {$[3,6,16,17]$} \\
\hline 29 & $\beta$-Pinene & Seed, fruit, aerial part & {$[3,4,6,13,15,17,23-25]$} \\
\hline 30 & Myrcene & Seed, fruit, aerial part & {$[4,6,15,17,24,25]$} \\
\hline 31 & 8-3-Carene & Seed, fruit & {$[6,17,25]$} \\
\hline 32 & o-cymene & Seed & {$[25]$} \\
\hline 33 & Limonene & Seed, fruit & {$[3,4,6,13,15-17]$} \\
\hline 34 & ${ }^{\text {-Terpinene }}$ & Seed, fruit & $8-4-2-[6,16,25]$ \\
\hline 35 & Terpinolene & Seed, fruit & {$[6,16,17,25]$} \\
\hline 36 & a-pinene oxide & Seed, fruit & {$[3,25]$} \\
\hline 37 & Bornyl acetate & Seed, fruit & {$[6,13,17]$} \\
\hline 38 & Eugenol & Fruit & {$[17]$} \\
\hline 39 & Perilla alcohol & Seed, fruit, aerial part & {$[3,6,13,15-17,24,25]$} \\
\hline 40 & Perillaldehyde & Seed, fruit, aerial part & {$[3,4,6,13,15-17,24,25]$} \\
\hline 41 & D-Limonene & Fruit, aerial part & {$[23,24]$} \\
\hline 42 & I-perrillaldehyde & Fruit & {$[23]$} \\
\hline 43 & 3-Thujanone & Aerial part & {$[24]$} \\
\hline 44 & p-cymene & Fruit & {$[3]$} \\
\hline
\end{tabular}


Table 1 Phytochemicals isolated from different parts of Ammodaucus leucotrichus (Continued)

\begin{tabular}{|c|c|c|c|}
\hline No. & Chemical compound & Part of plant & References \\
\hline$\overline{45}$ & Thymol & Fruit & [6] \\
\hline 46 & Cuminal & Seed & {$[25]$} \\
\hline 47 & a-Terpineol & Seed, fruit & {$[3,6,13,17]$} \\
\hline 48 & Terpen-1-ol & Fruit & {$[22]$} \\
\hline 49 & trans-Verbenol & Fruit & {$[17]$} \\
\hline 50 & trans-Carveol & Fruit & {$[17]$} \\
\hline 51 & a-Copaene & Seed, fruit & {$[6,13,25]$} \\
\hline 52 & $\beta$-Cubebene & Seed, fruit & {$[6,17,25]$} \\
\hline 53 & $\beta$-elemene & Seed & {$[25]$} \\
\hline 54 & Caryophyllene & Seed, aerial part & {$[13,24,25]$} \\
\hline 55 & a-caryophyllene & Seed & {$[25]$} \\
\hline 56 & a-selinene & Seed & {$[25]$} \\
\hline 57 & a-Muurolene & Seed, fruit & {$[6,17,25]$} \\
\hline 58 & 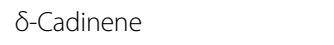 & Seed, fruit & {$[6,16,17,25]$} \\
\hline 59 & Spathulenol & Seed, fruit & {$[13,15,17,25]$} \\
\hline 60 & Caryophyllene oxide & Seed & {$[25]$} \\
\hline 61 & T-Muurolol & Seed, fruit & {$[6,25]$} \\
\hline 62 & a-Curcumene & Seed & [13] \\
\hline 63 & Bicyclogermacrene & Seed, fruit & {$[13,17]$} \\
\hline 64 & $\beta$-Dihydroagarofuran & Seed & [13] \\
\hline 65 & a-Cubebene & Fruit & {$[6]$} \\
\hline 66 & ar-Curcumene & Fruit & [6] \\
\hline 67 & a-Zingiberene & Fruit & [6] \\
\hline 68 & $\beta$-Bisabolene & Fruit & {$[6]$} \\
\hline 69 & $\gamma$-Cadinene & Fruit & {$[6,17]$} \\
\hline 70 & Germacrene B & Fruit & [6] \\
\hline 71 & $\beta$-Calacorene & Fruit & [6] \\
\hline 72 & Germacrene d-4-ol & Fruit & {$[6,17]$} \\
\hline 73 & 1-epi-Cubenol & Fruit & [6] \\
\hline 74 & Chamazulene & Fruit & [6] \\
\hline 75 & trans-a-Bergamotene & Fruit & {$[17]$} \\
\hline 76 & a-Humulene & Fruit & {$[17]$} \\
\hline 77 & $\beta$-Selinene & Fruit & {$[17]$} \\
\hline 78 & a-Bulnesene & Fruit & {$[17]$} \\
\hline 79 & 10-epi-Cubebol & Fruit & {$[17]$} \\
\hline 80 & T-Cadinol & Fruit & {$[17]$} \\
\hline 81 & $\beta$-Eudesmol & Fruit & {$[17]$} \\
\hline 82 & 11-Acetoxyeudesman-4-a-ol & Fruit & {$[17]$} \\
\hline 83 & $\beta$-Caryophyllene & Seed & [16] \\
\hline 84 & cis- $\beta$-Farnescene & Seed & [16] \\
\hline 85 & trans-Muurola-3,5-diene & Seed & [16] \\
\hline 86 & Germacrene D & Seed, fruit & {$[16,17]$} \\
\hline 87 & B -Selinene & Seed & [16] \\
\hline 88 & Globulol & Seed, aerial part & {$[16,24]$} \\
\hline
\end{tabular}


Table 1 Phytochemicals isolated from different parts of Ammodaucus leucotrichus (Continued)

\begin{tabular}{|c|c|c|c|}
\hline No. & Chemical compound & Part of plant & References \\
\hline 89 & a-Eudesmol & Seed, fruit & {$[16,17]$} \\
\hline 90 & Longifolol & Seed, fruit & {$[16,17]$} \\
\hline 91 & Ammolactone-A & Seed & {$[21]$} \\
\hline 92 & a-Cadinol & Seed & {$[6,13,17,25]$} \\
\hline 93 & a-Muurolol & Fruit & {$[6,17]$} \\
\hline 94 & Terpinen-4-ol & Seed, fruit & {$[6,17,25]$} \\
\hline 95 & 1-Pentadecene & Fruit & {$[23]$} \\
\hline 96 & 1-Nonadecene & Fruit & {$[23]$} \\
\hline 97 & Cuminaldehyde & Seed, aerial part, fruit & {$[4,13,16,17,24]$} \\
\hline 98 & a-Campholenal & Seed & [16] \\
\hline 99 & Benzaldehyde & Seed & {$[25]$} \\
\hline 100 & Allyl isovalerate & Seed, fruit & {$[16,17]$} \\
\hline 101 & $(Z, E)$-Farnesol & Fruit & [6] \\
\hline 102 & Methyl eugenol & Seed, fruit & {$[17,25]$} \\
\hline 103 & y-Decalactone & Seed, fruit & {$[6,17,25]$} \\
\hline 104 & cis-p-Mentha-2,8-dien-1-ol & Seed & {$[16]$} \\
\hline 105 & Allocimene B & Seed & {$[16]$} \\
\hline 106 & p-Cymen-8-ol & Seed & [16] \\
\hline 107 & cis-Pinocarveol & Seed & {$[16]$} \\
\hline 108 & trans-Isocarveol & Seed & [16] \\
\hline 109 & 11-Acetoxyeudesman-4-a-ol & Seed & {$[16]$} \\
\hline 110 & p-mentha-trans-2,8-dien-1-ol & Seed & {$[25]$} \\
\hline 111 & cis-limonene oxide & Seed & {$[16,25]$} \\
\hline 112 & Trans-p-mentha-1(7),8-dien-2-ol & Seed & {$[25]$} \\
\hline 113 & Carvacrol methyl ether & Fruit & {$[6]$} \\
\hline 114 & 3-Hydroxyperillaldehyde & Fruit & {$[6]$} \\
\hline 115 & 10-nor-Calamenen-10-one & Fruit & [6] \\
\hline 116 & a-Terpinen-7-al & Fruit & [4] \\
\hline 117 & y-Terpinen-7-al & Fruit & [4] \\
\hline 118 & Dehydro- Sabina ketone & Fruit & {$[17]$} \\
\hline 119 & Trans-p-Mentha-2,8-dien-1-ol & Fruit & {$[17]$} \\
\hline 120 & Cis-p-Mentha-2,8-dien-1-ol & Fruit & {$[17]$} \\
\hline 121 & Trans-Limonene oxide & Fruit & {$[17]$} \\
\hline 122 & Shybunol & Fruit & {$[17]$} \\
\hline 123 & Caryophyllene acetate & Fruit & {$[17]$} \\
\hline 124 & cis-p-mentha-trans-2,8-dien-1-ol & Fruit & {$[3]$} \\
\hline 125 & Pyranton & Fruit & [23] \\
\hline 126 & Rosifoliol & Fruit & [23] \\
\hline 127 & z-5-Nonadecene & Fruit & [23] \\
\hline 128 & a-copaen-11-ol & Aerial part & {$[24]$} \\
\hline 129 & |solongifolan-8-ol & Aerial part & {$[24]$} \\
\hline
\end{tabular}

aureus and Bacillus subtilis. The hydro-distilled oil it was inactive against Escherichia coli, Pseudomonas aeruginosa and Salmonella typhi [16]. Botrytis cinerea and Penicillium expansum showed a strong sensitivity to Ammodaucus leucotrichus essential oil at all concentrations [4]. 
The Essential oil showed in vitro, important antifungal activities against germination and sporulation spores. Aspergillus ochraceus was most sensitive, being inhibited at minimum sporulation inhibitory concentration as weak as $1 / 8000(\mathrm{v} / \mathrm{v})$. The Essential oil was found to be fungicidal at minimal fungicidal concentration against Aspergillus flavus [13].

The essential oil, acquired from the fruits were most active against Escherichia coli, Enterobacter cloaceae, Bacillus cereus, Salmonella typhimurium, Klebsiella pneumoniae and Staphylococcus aureus, and moderate active against Enterococcus faecium. The essential oil exhibited a strong antifungal activity against Fusarium oxysporum Aspergillus flavus, Candida albicans and Saccharomyces cerevisae strain [3, 23, 27]. The hydroethanolic extract and aqueous decoction from the aerial parts of Ammodaucus leucotrichus were moderately active against Gram-negative and Gram-positive bacteria. Both extracts obtained from the plant had no effect on Proteus mirabilis and very low effect on Klebsiella pneumoniae, even at the highest concentration tested $(20 \mathrm{mg} / \mathrm{mL})$ [14].

\section{Antidiabetic activities}

El-Ouady et al. [26] evaluated anti-diabetic activities of aqueous extract of Ammodaucus leucotrichus fruits in streptozotocin-induced diabetic rats. Blood glucose was significantly reduced after 15 days of oral administration of the plant extract at a dose of $10 \mathrm{mg} / \mathrm{kg}$. In addition, Ammodaucus leucotrichus fruit aqueous extract was able to exhibit a beneficial effect on histologlogical structure of liver and a remarkable influence on glucose tolerance [26].

\section{Anti-inflammatory activities}

The anti-inflammatory effect of the essential oil extracted from Ammodaucus leucotrichus fruits has been demonstrated using a dose of $100 \mathrm{mg} / \mathrm{kg}$ to evaluate the antiedematogenic response of essential oils in Carrageenaninduced hind paw edema in mice. The inhibitory activity of the essential oils was compared with that of diclofenac used as a standard drugs in this model. The mean increase in paw edema weight in control treated group mice (Group I) was $31.06 \pm 0.27 \mathrm{mg}$. The groups II, III, IV and $\mathrm{V}$ treated with essential oils have shown similar inhibition percentages (18.7\%, 21.6, 19.4 and 24.5\%) of Carrageenaninduced edema as compared with control group [3]. In another Study, Ziani et al. [14] assessed anti-inflammatory property of hydroethanolic extract from the aerial parts of Ammodaucus leucotrichus through the determination of the concentration of nitrite NO produced by lipopolysaccharide (LPS)-stimulated murine macrophages RAW 264.7 cell lines in culture medium, treated with different concentrations of the plant extracts. The plant extracts, effectively reduced the expression of ant-inflammatory enzymes iNOS in LPS-activated murine macrophages, and this reduction was accompanied by the decrease of the nitric oxide (NO) levels [14].

\section{Anticholinesterase activities}

The anticholinesterase activity of the Ammodaucus leucotrichus and the individual main compounds of the essential oil were compared to Donepezil, used as a standard drug against Alzheimer's disease. Essential oil and perillaldehyde have not exhibited an activity against the acetylcholinesterase. In contrast, limonene induced high acetylcholinesterase inhibitory activity with an IC50 of about $51.6 \mu \mathrm{g} / \mathrm{mL}$. Essential oil appeared to be effective compared to inhibition results of Donepezil. For anti-butyrylcholinesterase activity, all samples have a high inhibitory activity and the lowest value of IC50 was obtained with perillaldehyde followed by limonene and essential oil [24].

\section{Cytotoxicity activities}

Ziani et al. [14] evaluated cytotoxicity activities of aqueous decoction and aerial parts hydroethanolic extracts of Ammodaucus leucotrichus. The inhibitory growth activity of four human tumor cell lines: NCI-H460 (nonsmall cell lung cancer), HeLa (cervical carcinoma), HepG2 (hepatocellular carcinoma), and MCF-7 (breast carcinoma), was determined by the sulforhodamine $\mathrm{B}$ colorimetric assay. The hepatotoxicity was determined using a primary culture of nontumor liver cells (PLP2), which were prepared from a freshly harvested porcine liver. The GI50 values for the hydroethanolic extract were in general lower than the ones exhibited by decoction extracts, showing a significant dose dependent cytotoxic effect. The cell lines MCF-7, HeLa, and NCI-H460 were the most susceptible for the hydroethanolic extract of Ammodaucus leucotrichus [14].

\section{Conclusions}

The available literature on Ammodaucus leucotrichus has shown that it is an important medicinal plant used in a wide range of ethnomedical treatments, especially for diarrhea, cough, pulmonary diseases, anorexia, allergy, tachycardia, helminthiasis, gastric-intestinal pains, gastralgias, otitis, indigestion, cold, fever, pains of tiredness, anorexia and cardiac diseases. Monoterpenes and their derivatives, sesquiterpenes and their derivatives, tannins, anthracenes compounds, sterols, triterpenes, reducing compounds, alkaloids, phenol acids, saponins, flavonoids and coumarins are the major chemical constituents which have been demonstrated in Ammodaucus leucotrichus. Studies have shown that various extracts of Ammodaucus leucotrichus possess a range of pharmacological actions, such as antioxidant, antibacterial, antifungal, antidiabetic, anti-inflammatory, anticholinesterase and cytotoxicity activities supporting its 
traditional use. However, the most prominent and the well studied activities are the antimicrobial and antioxidant activities of Ammodaucus leucotrichus extracts, fractions and secondary metabolites isolated from various parts in different experimental models. Therefore, considering its versatile medicinal uses. Further improvements are required to encourage research interest on Ammodaucus leucotrichus.

\section{Abbreviations}

BHT: Butylated hydroxytoluene; DPPH: 2,2-Diphenyl-1-picrylhydrazyl;

IC50: Minimum inhibition concentration for inhibiting 50\% of the pathogen; LPS: Lipopolysaccharide; MIC: Minimum inhibitory concentration; NO: Nitric oxide

\section{Acknowledgements}

Not applicable.

\section{Authors' contributions}

El Manuscript preparation. FM Manuscript review. KC Supervising the whole work. All authors read and approved the final manuscript.

\section{Funding}

There is no funding for review article.

Availability of data and materials

Not applicable.

Ethics approval and consent to participate

Not applicable.

\section{Consent for publication}

Not applicable.

\section{Competing interests}

The authors declare that they have no competing interests.

Received: 4 May 2019 Accepted: 27 January 2020

Recrived: 4 May 2019 Accepted: 27 January

\section{References}

1. Ouhaddou H, Boubaker H, Msanda F, El Mousadik A. An ethnobotanical study of medicinal plants of the Agadir Ida Ou Tanane province (Southwest Morocco). J Appl Biosci. 2014;84(1):7707-22.

2. Manssouri M, El Ouadi Y, Znini M, Costa J, Bouyanzer A, Desjobert J, Majidi L. Adsorption proprieties and inhibition of mild steel corrosion in $\mathrm{HCl}$ solution by the essential oil from fruit of Moroccan Ammodaucus leucotrichus. J Mater Environ Sci. 2015;6(3):631-46.

3. Mohammedi H, Idjeri-Mecherara S, Menaceur F, Azine K, Hassani A. Chemical compositions of extracted volatile oils of Ammodaucus leucotrichus L. fruit from different geographical regions of Algeria with evaluation of its toxicity, anti-inflammatory and antimicrobial activities. J Essent Oil Bear PI. 2018;21(6):1568-84

4. Manssouri M, Znini M, El Harrak A, Majidi L. Antifungal activity of essential oil from the fruits of Ammodaucus leucotrichus Coss. \& Dur., in liquid and vapour phase against postharvest phytopathogenic fungi in apples. J Essent Oil Bear PI. 2016;6(05):131-6.

5. Sebaa A, Marouf A, Kambouche N, Derdour A. Phytochemical composition, antioxidant and antimicrobial activities of Ammodaucus leucotrichus fruit from Algerian Sahara. Orient J Chem. 2018;34(1):519.

6. Velasco-Negueruela A, Perez-Alonso M, De Paz PP, Pala-Paul J, Sanz J. Analysis by gas chromatography-mass spectrometry of the volatiles from the fruits of Ammodaucus leucotrichus subsp. Leucotrichus and subsp. nanocarpus grown in North Africa and the Canary Islands, respectively. J Chromatogr A. 2006;1108(2):273-5.

7. El Hafian M, Benlandini N, Elyacoubi H, Zidane L, Rochdi A. Étude floristique et ethnobotanique des plantes médicinales utilisées au niveau de la préfecture d'Agadir-lda-Outanane (Maroc). J Appl Biosci. 2014;81(1):7198-213.
8. Bouayyadi L, El Hafian M, Zidane L. Étude floristique et ethnobotanique de la flore médicinale dans la région du Gharb, Maroc. J Appl Biosci. 2015;93: 8770-88.

9. Tahri N, El Basti A, Zidane L, Rochdi A, Douira A. Etude ethnobotanique des plantes medicinales dans La province De Settat (Maroc). Kastamonu Univ Orman Fak Derg. 2012;12(2):192-208.

10. Benkhnigue O, Hachi M, Fadli M, Douira A, Zidane L. Catalogue of the medicinal plants used in the treatment of urinary infections in the area of al-Haouz rhamna (Central Morocco). Eur J Bot Plant Sci Phyt. 2016;3:1-49.

11. Ghourri M, Zidane L, Douira A. Catalogue des plantes médicinales utilisées dans le traitement de la lithiase rénale dans la province de Tan-Tan (Maroc saharien). Int J Biol Chem Sci. 2013;7(4):1688-700.

12. Abouri M, El Mousadik A, Msanda F, Boubaker H, Saadi B, Cherifi K. An ethnobotanical survey of medicinal plants used in the Tata Province, Morocco. Int J Med Plants Res. 2012;1(7):99-123.

13. Khaldi A, Meddah B, Moussaoui A, Sonnet P. Anti-mycotoxin effect and antifungal properties of essential oil from Ammodaucus leucotrichus Coss. \& Dur. On Aspergillus flavus and Aspergillus ochraceus. J Essent Oil Bear PI. 2017:20(1):36-44.

14. Ziani BE, Rached W, Bachari K, Alves MJ, Calhelha RC, Barros L, Ferreira IC. Detailed chemical composition and functional properties of Ammodaucus leucotrichus cross. \& Dur. And Moringa oleifera Lamarck. J Funct Foods. 2019;53:237-47.

15. Halla N, Heleno SA, Costa P, Fernandes IP, Calhelha RC, Boucherit K, Rodrigues AE, Ferreira IC, Barreiro MF. Chemical profile and bioactive properties of the essential oil isolated from Ammodaucus leucotrichus fruits growing in Sahara and its evaluation as a cosmeceutical ingredient. Ind Crop Prod. 2018;119:249-54.

16. Abu Zarga MH, Al-Jaber HI, Baba Amer ZY, Sakhrib L, Al-Qudah MA, Alhumaidi JY, Abaza IF, Afifi FU. Chemical composition, antimicrobial and antitumor activities of essential oil of Ammodaucus leucotrichus growing in Algeria. J Biol Act Prod Nat. 2013;3(3):224-31.

17. Dahmane D, Dob T, Krimat S, Nouasri A, Metidji H, Ksouri A. Chemical composition, antioxidant and antibacterial activities of the essential oils of medicinal plant Ammodaucus leucotrichus from Algeria. J Essent Oil Res. 2017;29(1):48-55.

18. Bahramsoltani R, Rostamiasrabadi P, Shahpiri Z, Marques AM, Rahimi R, Farzaei MH. Aloysia citrodora Paláu (lemon verbena): a review of phytochemistry and pharmacology. J Ethnopharmacol. 2018;222:34-51.

19. Peng W, Liu Y-J, Wu N, Sun T, He X-Y, Gao Y-X, Wu C-J. Areca catechu L. (Arecaceae): a review of its traditional uses, botany, phytochemistry, pharmacology and toxicology. J Ethnopharmacol. 2015;164:340-56.

20. Shakeri A, Sahebkar A, Javadi B. Melissa officinalis L.-a review of its traditional uses, phytochemistry and pharmacology. J Ethnopharmacol. 2016;188:204-28.

21. Muckensturm B, Diyani F, Le Nouën D, Fkih-Tetouani S, Reduron J-P. Ammolactone, a guaianolide from a medicinal plant, Ammodaucus leucotrichus. Phytochemistry. 1997:44(5):907-10.

22. Moulay A, Brahim S, Satrani B, Ghanmi M, Aafi A, Amusant N, El Antry S, Chaouch A. Bioactivity and chemical quality of Ammodaucus leucotrichus ssp. Leucotrichus Coss. \& Durieu essential oils from Morocco. Nat Prod. 2014;10(6):208-14.

23. Gherraf N, Zellagui A, Kabouche A, Lahouel M, Salhi R, Rhouati S. Chemical constituents and antimicrobial activity of essential oils of Ammodaucus leucotricus. Arab J Chem. 2017;10:S2476-8.

24. Sadaoui N, Bec N, Barragan-Montero V, Kadri N, Cuisinier F, Larroque C, Arab K, Khettal B. The essential oil of Algerian Ammodaucus leucotrichus Coss. \& Dur. And its effect on the cholinesterase and monoamine oxidase activities. Fitoterapia. 2018;130:1-5.

25. Louail Z, Kameli A, Benabdelkader T, Bouti K, Hamza K, Krimat S. Antimicrobial and antioxidant activity of essential oil of Ammodaucus leucotrichus Coss. \& Dur. Seeds. J Mater Environ Sci. 2016;7(7):2328-34

26. El-Ouady F, Eddouks M. Glucose lowering activity of aqueous Ammodaucus leucotrichus extract in diabetic rats. Cardiovasc Haematol Disord Drug Targets. 2019;19:1-8.

27. El-Haci IA, Bekhechi C, Atik-Bekkara F, Mazari W, Gherib M, Bighelli A, Casanova J, Tomi F. Antimicrobial activity of Ammodaucus leucotrichus fruit oil from Algerian Sahara. Nat Prod Commun. 2014;9(5):711-2.

\section{Publisher's Note}

Springer Nature remains neutral with regard to jurisdictional claims in published maps and institutional affiliations. 